\title{
Synthesis and tunable emission of novel polyfluorene co-polymers with 1,8 - naphthalimide pendant groups and application in a single layer-single component white emitting device
}

Carmen Coya ${ }^{\mathrm{c}}$, Raúl Blanco ${ }^{\mathrm{a}}$, Rafael Juárez ${ }^{\mathrm{a}}$,, Rafael Gómez $^{\mathrm{a}}$, Rocío Martínez ${ }^{\mathrm{d}}$, Alicia de Andrés ${ }^{\mathrm{d}}$, Ángel Luis Álvarez ${ }^{\mathrm{e}}$, Carlos Zaldo ${ }^{\mathrm{d}}$, María M. Ramos ${ }^{\mathrm{b}}$, Alejandro de la Peña ${ }^{\mathrm{a}}$, Carlos Seoane ${ }^{\mathrm{a}}$, José L. Segura ${ }^{\mathrm{a}}$

a Departamento de Química Orgánica, Facultad de Ciencias Químicas, Universidad Complutense de Madrid, E-28040 Madrid, Spain

b Departamento de Tecnología Química y Ambiental, Escuela Superior de Ciencias Experimentales y Tecnología, Universidad Rey Juan Carlos, E-28933 Móstoles, Spain

c Área de Electrónica, Departamento de Teoría de la Señal y Comunicaciones, Escuela Superior de Ingeniería de Telecomunicación, Universidad Rey Juan Carlos, E-28943 Fuenlabrada, Spain

d Instituto de Ciencia de Materiales, CSIC, Cantoblanco, 28049 Madrid, Spain

e Departamento de Tecnología Electrónica, Escuela Superior de Ciencias Experimentales y Tecnología, Universidad Rey Juan Carlos, E-28933 Madrid, Spain

Versión final:

European Polymer Journal, Volume 46, Issue 8, August 2010, Pages 1778-1789

DOI: 10.1016/j.eurpolymj.2010.04.020

http://dx.doi.org/10.1016/j.eurpolymj.2010.04.020 


\begin{abstract}
New luminescent polymers containing two individual emission species-poly(fluorene-altphenylene) as a blue host and variable amounts of 1,8-naphthalimide as red dopant have been designed and synthesized. Optical studies (optical absorption (OA) and steady-state photoluminescence emission (PL)) in diluted solutions and thin solid films reveal that the emission spectrum can be tuned by varying the content of 1,8-naphthalimide moieties. Although no significant interaction can be observed between both moieties in the ground state, after photoexcitation an efficient energy transfer takes place from the PFP backbone to the red chromophore, indeed, by adjusting the polymer/naphthalimide ratio it is possible to obtain single polymers which emit white light to the human eye in solid state. Energy transfer is more effective in the co-polymers than in physical mixtures of the two chromophores. We prepared single-layer electroluminescent simple devices with structure: ITO/poly(3,4ethylenedioxythiophene)/poly(4-styrenesulfonate) (PEDOT:PSS)/active layer/Ba/Al. With this single layer-single component device structure, white light with Commission Internationale de I'Eclairage (CIE) color coordinates $(0.3,0.42)$ is obtained for the electroluminescence (EL) emission with an efficiency of $22.62 \mathrm{Cd} / \mathrm{A}$.
\end{abstract}

\title{
1. Introduction
}

A great deal of effort has been devoted in recent years to the design and synthesis of conjugated polymers functionalized with different groups with the aim to obtain materials with specific physical and chemical properties. This approach has been explored for the preparation of materials with exciting properties and applications, such as sensors [1] or energy conversion materials [2], [3] and [4]. In the latter regard, semiconducting polymers substituted with chromophores and exhibiting photoinduced electron and/or energy transfer have been synthesized as promising candidates for the preparation of enhanced devices [4]. Thus, we and others have synthesized and investigated the photophysical behaviour of semiconducting polymer backbones carrying chromophores such as fullerenes [5], tetracyanoanthraquinodimethane [6], [7] and [8], anthraquinone [9], [10] and [11] or perylenediimide (PDI) as the pendant groups [12], [13] and [14]. An active area of research involves the development of single polymers with simultaneous blue and orange emission, in which a small amount of orange light-emissive derivatives are incorporated into the main chain of a blue light emitting polymer with the aim to obtain white luminescent materials for light emitting diodes [15], [16], [17] and [18].

White polymer light emitting diodes (WPLEDs) have attracted particular research interest owing to their possible use in full-color displays combined with a color filter, such as backlights for liquid-crystal displays or other lighting applications [19]. In fact, as they are solution processed, these materials have become an strategic issue in solid-state lighting (SSL) for their 
potential energy saving, thin, flexible WOLEDs to replace traditional incandescent white light sources in large area displays [20].

A variety of methods have been proposed to achieve white-light-emitting diodes. One approach to produce white-light-emission has been the use of a multilayer device system consisting of two or more active layers, where each layer emitted a primary color [21]. However, multilayer devices fabricated by vapour deposition were difficult to assemble [22]. On the other hand, simultaneously, several approaches using polymer blend systems, such as three-polymer blends containing red-, green- and blue-light-emitting polymers and twopolymer blends containing blue- and orange-light-emitting polymers [23] have been investigated. Heeger's group reported white electroluminescence by combination of two fluorescent polymers blended with an organometallic complex [24]. The problem found in this kind of single-layer device was color instability due to phase separation of the different polymer components. Using a single polymer as the emissive layer could solve the problem of phase separation in blend systems. Thus, compared to the multi-emitting-component WOLEDs, a single-emitting-component WPLED could show many advantages, such as better stability, better reproducibility, and simpler fabrication process. Therefore, the search for new organic light-emitting materials with new structures for use in single-emitting-component WOLEDs is of obvious interest and importance.

In this work we report a new synthetic route toward blue emitting poly(fluorene-altphenylene) (PFP) derivatives containing comonomers bearing red-emitting 1,8-naphthalimide units as pendant groups (P1-P4, Fig. 1). The naphthalimide dopant has been covalently attached to the pendant chain of the host with an alkyl spacer to realize molecular dispersion of the dopant. We demonstrate that this strategy prevents phase separation and the formation of aggregates and favours energy transfer in the compound, allowing the tuning of the emission by tailoring the content of the naphthalimide dopant. We have investigated the photophysical behaviour of the novel polymers by means of optical absorption (OA) and steady-state photoluminescence (PL), PL decay and we have tested their device properties in single-component, single-layer organic light emitting diodes (OLEDs).

\section{Experimental}

Compounds 1-hexyloxy-2,5-diiodo-4-(6'-iodohexyloxy)-benzene (3) [25], 4-(phenyl-2naphthyl)amino-1,8-naphthalic anhydride (1) [13], 4-(1-naphthylphenylamine)-N-phenyl-1,8naphthalimide (7) [26], 2,7-bis(4,4,5,5-tetramethyl-1,3,2-dioxoborolan-2-yl)-9,9dioctylfluorene (5) [27], 1,4-dihexyloxy-2,5-diiodobenzene (6) [28] and poly[9,9-dioctyl-9Hfluorene-2,7-diyl][2,5-bis(hexyloxy)-1,4-phenylene] (PFP) [29] were prepared according to the literature. All other chemicals were purchased from Aldrich and used as received without further purification. Column chromatography was performed on Merck Kieselgel 60 silica gel (230-240 mesh). Thin layer chromatography was carried out on Merck silica gel F-254 flexible TLC plates. Solvents and reagents were dried by usual methods prior to use and typically used under inert gas atmosphere.

\subsection{Characterization}


Melting points were measured with an electrothermal melting point apparatus and are uncorrected. FTIR spectra were recorded as $\mathrm{KBr}$ pellets in a Shimadzu FTIR 8300 spectrometer. NMR were recorded on a Bruker AC-200, Avance 300 or AMX-400 apparatus as noted, and the chemical shifts were reported relative to tetramethylsilane (TMS) at $0.0 \mathrm{ppm}$ (for ${ }^{1} \mathrm{H} N M R$ ) and $\mathrm{CDCl}_{3}$ at $77.16 \mathrm{ppm}$ (for ${ }^{13} \mathrm{C} \mathrm{NMR}$ ). The splitting patterns are designated as follows: $\mathrm{s}$ (singlet), $\mathrm{d}$ (doublet), $\mathrm{t}$ (triplet), $\mathrm{m}$ (multiplet) and $\mathrm{q}$ (quadruplet). Mass spectra were recorded with a Varian Saturn 2000 GC-MS and with a MALDI-TOF MS Bruker Reflex 2 (dithranol as matrix). Elemental analyses were performed on a Perkin-Elmer EA 2400. Average molecular weights $\overline{M_{w}}$ and $\overline{M_{n}}$ and molecular weight distribution were determined by gel permeation chromatography (GPC) in a Aliance 2000 Waters GPC coupled with refractive index (RI) and viscosity detectors. The solvent used for the analysis was 1,2,4,5-tetrachlorobenzene (TCB), the flow rate was $1.0 \mathrm{~mL} / \mathrm{min}$ and the temperature was $145^{\circ} \mathrm{C}$. The GPC-viscosity system was calibrated using polystyrene standards.

\subsection{Electrochemistry}

Cyclic voltammetry experiments were performed with a computer controlled EG \& G PAR 273 potentiostat in a three-electrode single-compartment cell $(5 \mathrm{ml})$. The platinum working electrode consisted of a platinum wire sealed in a soft glass tube with a surface of $A=0.785 \mathrm{~mm}^{2}$, which was polished down to $0.5 \mu \mathrm{m}$ with Buehler polishing paste prior to use in order to obtain reproducible surfaces. The counter electrode consisted of a platinum wire and the reference electrode was a $\mathrm{Ag} / \mathrm{AgCl}$ secondary electrode. All potentials were internally referenced to the ferrocene-ferrocinium couple. For the measurements, concentrations of $5 \times 10^{-3} \mathrm{~mol} \mathrm{I}^{-1}$ of the electroactive species were used in freshly distilled and deaerated dichloromethane (Lichrosolv, Merck) and $0.1 \mathrm{M}$ tetrabutylammonium perchlorate $\left(\mathrm{TBAClO}_{4}\right.$, Fluka) which was twice recrystallized from ethanol and dried under vacuum prior to use.

\subsection{Devices fabrication}

The structure of the devices are ITO/PEDOT:PSS $(50 \mathrm{~nm}) /$ active layer $(91-123 \mathrm{~nm}) / \mathrm{Ba}$ $(20 \mathrm{~nm}) /$ Al. Pre-patterned ITO glass plates were extensively cleaned, using chemical and UVozone methods, just before the deposition of the organic layers. The thickness of the layers were measured using an Alpha step 200 profilomenter (Tenkor Instruments). The active layers were deposited by spin coating from chloroform $\left(\mathrm{CHCl}_{3}\right)$ solutions $(10 \mathrm{mg} / \mathrm{ml})$. The resulting layers thickness was: 121, 91, 123, 112 and 91 nm for PFP, P1, P2, P3 and P4, respectively. We have found that heating the sample just below the solvent boiling point reduces aggregation and results in an improved film uniformity (highly reflecting surfaces) [30]. The Ba and Al cathode was thermally evaporated in an atmosphere of $5 \times 10^{-5}$ Torr on top of the organic layer surface and the structure is encapsulated using a glass cover attached by a bead of epoxy adhesive [EPO-TEK(302-3 M)]. All the process is carried out in an inert atmosphere glovebox ( $<0.1$ ppm $\mathrm{O}_{2}$ and $\mathrm{H}_{2} \mathrm{O}$ ).

Thin films of P1-P4 and PFP on quartz substrates were fabricated by spin coating from $10 \mathrm{mg} / \mathrm{ml}$ chloroform precursor solutions to investigate the optical properties in thin film. Also a thin film of a physical blend (Blend) between PFP and the naphthalimide reference 7 was fabricated with the same polymer/naphthalimide ratio as in P4 (i.e. 0.08) for comparison porpouses. 


\subsection{Optical measurements}

The OA and PL spectra of the material in solution and in thin films on quartz substrates have been measured at room temperature. UV-vis OA of solutions and PL spectra for both, solutions and films, were recorded with a Perkin-Elmer Lambda 900 spectrophotometer and an Edinburgh Instruments FS920 double-monochromator luminescence spectrometer using a Peltier-cooled red-sensitive photomultiplier, respectively. The OA thin film spectra were recorded with a Varian spectrophotometer model Cary 4000. Spectral luminance from diodes was recorded with a Konica-Minolta CS-2000 spectroradiometer, in the same excitation conditions (duty cycle) as those used to measure $I-V$ characteristics (duty cycle of $0.2 \%$ ). The $\mathrm{PL}$ quantum efficiencies of polymer were estimated by using a thin film of perylene as blue standard $\left(\phi_{\mathrm{PL}}=0.87\right)[31]$.

$\phi_{s}=\phi_{r} \frac{F_{s}}{F_{r}} \frac{A_{r}}{A_{s}}\left(\frac{n_{r}}{n_{s}}\right)^{2}$

In the above expression, $\phi$ is the fluorescent quantum yield, $F$ the integrated emission intensity, $n$ the refractive index, and $A$ the absorbance at the exciting wavelength. The subscripts $r$ and $s$ denote the reference and unknown samples, respectively.

The PL lifetimes were recorded at room temperature. The excitation was made at $380 \mathrm{~nm}$ with a dye laser using PBBO. This laser provides pulses with $10 \mu \mathrm{J}$ of energy and a FWHM of $5 \mathrm{~ns}$, however it also radiates a 50 ns delayed pulse replica with intensity 25 times lower than the main pulse, this compromises the analysis of the long time tails of the light decays. The emission dispersed by an SPEX spectrometer $(f=34 \mathrm{~cm})$ was recorded with a Hamamatsu R2658 cooled photomultiplier and the signal is stored and analyzed in a digital $500 \mathrm{MHz}$ Tektronix oscilloscope. To remove straight light from the excitation laser long-wavelength pass filters were set before the photomultiplier window; nevertheless some light contamination was always present. Since the time decays of the signals analyzed were below 20 ns the contribution of the excitation light scattered by the samples was evaluated and discounted from the photoluminescence signals.

\subsection{Electrical measurements}

The device current-voltage characteristics were measured using a semiconductor parameter analyzer Agilent 4155C and a SMU pulse generator Agilent 41501B. A pulse train was used as input signal. The duty cycle was set to be $0.2 \%$, thus having a pulse width of $0.5 \mathrm{~ms}$ for a period of $100 \mathrm{~ms}$. Refresh time between two consecutive pulses ensures long time operation without significant device degradation. Furthermore, the $I-V$ curve stability was achieved by gradually increasing the pulse amplitude up to the point where reproducible measurements were observed. The voltage range is below the onset for the electroluminescence (EL).

\subsection{Synthesis of 6-(1-naphthalenylphenylamino)-2-(4-hydroxyphenyl)-1H-benz[de]- isoquinoline-1,3(2H)-dione (2)}

Under argon atmosphere, 4-(phenyl-2-naphthyl)amino-1,8-naphthalic anhydride (1) (100 mg, $0.24 \mathrm{mmol})$ and 4-aminophenol $(105 \mathrm{mg}, 0.96 \mathrm{mmol})$ were mixed with imidazole $(7 \mathrm{~g})$ and 
heated at $180^{\circ} \mathrm{C}$ for $4 \mathrm{~h}$. After cooling, the solid was disolved in dichloromethane $(30 \mathrm{ml})$ and washed with a concentrated solution of $\mathrm{HCl}$ three times $(\times 30 \mathrm{ml})$ and then with brine. The organic phase was dried over $\mathrm{MgSO}_{4}$, filtered and vacuum evaporated. The remaining residue was purified by column chromatography (silica gel, dichloromethane/methanol 99/1) to yield compound $\mathbf{2}$ in $73 \%$ yield as a red-orange solid.

M.p.: $182-184^{\circ} \mathrm{C}$ (dichloromethane/methanol).

${ }^{1} \mathrm{H}$ NMR ( $\left.\mathrm{CDCl}_{3}, 400 \mathrm{MHz}\right) .8 .59(\mathrm{dd}, J=7.26 \mathrm{~Hz}, J=1.00 \mathrm{~Hz}, 1 \mathrm{H}), 8.58(\mathrm{~d}, J=8.00 \mathrm{~Hz}, 1 \mathrm{H}), 8.28$ (dd, $J=8.55 \mathrm{~Hz}, J=1.00 \mathrm{~Hz}, 1 \mathrm{H}), 7.79(\mathrm{~d}, J=9.00 \mathrm{~Hz}, 1 \mathrm{H}), 7.78(\mathrm{~d}, J=8.80 \mathrm{~Hz}, 1 \mathrm{H}), 7.59-7.39$ $(\mathrm{m}, 5 \mathrm{H}), 7.37-7.27(\mathrm{~m}, 5 \mathrm{H}), 7.14-7.08(\mathrm{~m}, 4 \mathrm{H}), 6.89-6.80(\mathrm{~m}, 2 \mathrm{H}), 6.46(\mathrm{br}, 1 \mathrm{H})$.

${ }^{13} \mathrm{C} \mathrm{NMR}\left(\mathrm{CDCl}_{3}, 100 \mathrm{MHz}\right) .165 .0$ (C=0), $164.4(\mathrm{C}=0), 156.3,151.3,148.2,145.9,134.2$, $132.8,131.9,131.7,130.7,130.4,129.7,129.6,128.3,128.0,127.6,127.3,127.1,126.7,126.5$, $125.6,125.2,124.1,123.6,123.3,120.5,118.7,116.7$.

$\mathrm{FTIR}(\mathrm{KBr}) \cdot v=3360,3075,1704,1657,1582,1491,1368,754 \mathrm{~cm}^{-1}$.

MS (FAB) $(m / z) 507\left([M+1]^{+}\right)$.

Anal. calc. for $\mathrm{C}_{34} \mathrm{H}_{22} \mathrm{~N}_{2} \mathrm{O}_{3}$ : C: $80.62 \%$; $\mathrm{H}: 4.38 \%$; N: $5.53 \%$. Found: C: $80.70 \%$; $\mathrm{H}: 4.36 \%$; : $5.60 \%$.

\subsection{Synthesis of 6-(1-naphthalenylphenylamino)-2-[4-[[6-[4-(hexyloxy)-2,5-diiodo- phenoxy]hexyl]oxy]phenyl]-1H-benz[de]isoquinoline-1,3(2H)-dione (4)}

Under an argon atmosphere, a mixture of 1-hexyloxy-2,5-diiodo-4-(6'-iodohexyloxy)benzene (3) $\quad(150 \mathrm{mg}, \quad 0.23 \mathrm{mmol}), \quad 6$-(1-naphthalenylphenylamino)-2-(4-hydroxyphenyl)-1Hbenz[de]isoquinoline-1,3(2H)-dione (2) $(151 \mathrm{mg}, 0.30 \mathrm{mmol})$ and potassium carbonate (124 mg, $0.90 \mathrm{mmol}$ ) was heated at $100^{\circ} \mathrm{C}$ in $25 \mathrm{ml}$ of anhydrous $\mathrm{N}, \mathrm{N}$-dimethylformamide for $48 \mathrm{~h}$. After this time, the crude was allowed to reach room temperature and treated with a $1 \mathrm{M}$ aqueous solution of hydrochloric acid. The mixture was extracted with dichloromethane and the organic extracts dried over anhydrous magnesium sulphate and then evaporated under vacuum. The residue was purified by flash chromatography (silica gel, hexane/dichloromethane $2 / 8$ ) to yield monomer 3 in $32 \%$ yield as an orange solid.

M.p.: $84-85^{\circ} \mathrm{C}$ (hexane/dichloromethane).

${ }^{1} \mathrm{H}$ NMR ( $\left.\mathrm{CDCl}_{3}, 200 \mathrm{MHz}\right) . \delta=8.59-8.54(\mathrm{~m}, 2 \mathrm{H}, \mathrm{Napht}), 8.25$ (dd, $J=8.6 \mathrm{~Hz}, J=1.22 \mathrm{~Hz}, 1 \mathrm{H}$, Napht), 7.82-7.75 (m, 2H, Napht), 7.59-7.03 (m, 18H), 4.01-3.90 (m, 6H, $\left.-\mathrm{OCH}_{2}{ }^{-}\right), 1.90-1.76$ $\left(\mathrm{m}, 6 \mathrm{H},-\mathrm{CH}_{2}{ }^{-}\right), 1.37-1.25\left(\mathrm{~m}, 10 \mathrm{H},-\mathrm{CH}_{2}{ }^{-}\right), 0.91\left(\mathrm{t}, 3 \mathrm{H},-\mathrm{CH}_{3}\right)$.

${ }^{13} \mathrm{C} \mathrm{NMR}\left(\mathrm{CDCl}_{3}, 125 \mathrm{MHz}\right) . \delta=164.70(\mathrm{C}=0), 164.13(\mathrm{C}=0), 159.09,152.92,152.83$, $151.04,148.34,145.95,134.26,132.55,131.74,131.47,130.75,130.39,129.75,129.60$, $129.54,128.45,128.17,128.13,127.79,127.72,127.45,127.13,126.88,126.75,126.54$, $125.74,125.21,124.12,124.03,123.65,123.50,122.81,120.49,119.07,115.26,86.38\left(C_{A r}-1\right)$, $86.34\left(\mathrm{C}_{\mathrm{Ar}} \mathrm{I}\right), 70.37\left(\mathrm{O}-\mathrm{CH}_{2}\right), 70.19\left(\mathrm{O}-\mathrm{CH}_{2}\right), 68.05\left(\mathrm{CH}_{2}-\mathrm{O}\right), 30.23,29.75,29.42,29.23,25.82$, $25.75,25.46,22.64,14.10$. 
FTIR $(\mathrm{KBr}) . v=2926,2855,1707,1668,1586,1511,1490,1465,1365,1241,1212,1175$, $855 \mathrm{~cm}^{-1}$.

$\operatorname{MS}(\mathrm{FAB})(\mathrm{m} / \mathrm{z}): 1036\left([\mathrm{M}+1]^{+}\right)$.

Anal. calcd. for $\mathrm{C}_{52} \mathrm{H}_{48} \mathrm{I}_{2} \mathrm{~N}_{2} \mathrm{O}_{5}$ : C: $60.36 \% ; \mathrm{H}: 4.68 \% ; \mathrm{N}: 2.71 \%$. Found: $\mathrm{C}: 60.49 \% ; \mathrm{H}: 4.61 \% ; \mathrm{N}$ : $2.55 \%$.

\subsection{Polymers P1-P4}

General procedure for polymerizations. Under an argon atmosphere, a solution of 2,7bis(4,4,5,5-tetramethyl-1,3,2-dioxoborolan-2-yl)-9,9-dioctylfluorene (4), 1,4-dihexyloxy-2,5diiodobenzene (5), monomer 6 and tetrakis(triphenylphosphine)palladium (0) in a deareated mixture of THF and an aqueous $2 \mathrm{M}$ potassium carbonate solution was heated at $100{ }^{\circ} \mathrm{C}$ for $48 \mathrm{~h}$. The mixture was allowed to reach the room temperature and then poured into methanol. The precipitate was collected by filtration, dissolved in dichloromethane and precipitated out of methanol. After soxhlet extraction with cloroform for $48 \mathrm{~h}$, the polymer was further purified by re-precipitation from dichloromethane/methanol several times. The corresponding polymers were isolated by centrifugation and dried under vacuum. The products were obtained after drying in vacuum with yields between $52 \%$ and $70 \%$.

P4: 2,7-Bis(4,4,5,5-tetramethyl-1,3,2-dioxoborolan-2-yl)-9,9-dioctylfluorene (4) (150 mg, $0.23 \mathrm{mmol}), 1,4$-dihexyloxy-2,5-diiodobenzene (5) $(112.0 \mathrm{mg}, 0.2116 \mathrm{mmol}$ ) and monomer 3 $(19.0 \mathrm{mg}, 0.0184 \mathrm{mmol})$ were used in the polymerization. GPC: $M_{n}=1.44 \times 10^{4}, \mathrm{PDI}=3.26$.

P3: 2,7-Bis(4,4,5,5-tetramethyl-1,3,2-dioxoborolan-2-yl)-9,9-dioctylfluorene (4) (150 mg, $0.23 \mathrm{mmol}$ ), 1,4-dihexyloxy-2,5-diiodobenzene (5) (119.0 $\mathrm{mg}, 0.2254 \mathrm{mmol}$ ) and monomer 3 $\left(4.76 \mathrm{mg}, 4.60 \times 10^{-3} \mathrm{mmol}\right)$ were used in the polymerization. GPC: $M_{n}=1.45 \times 10^{4}, \mathrm{PDI}=2.75$.

P2: 2,7-Bis(4,4,5,5-tetramethyl-1,3,2-dioxoborolan-2-yl)-9,9-dioctylfluorene (4) (150 mg, $0.23 \mathrm{mmol}$ ), 1,4-dihexyloxy-2,5-diiodobenzene (5) (121.3 $\mathrm{mg}, 0.2288 \mathrm{mmol}$ ) and monomer 3 $\left(1.19 \mathrm{mg}, 1.15 \times 10^{-3} \mathrm{mmol}\right)$ were used in the polymerization. GPC: $M_{n}=1.41 \times 10^{4}, \mathrm{PDI}=2.62$.

P1: 2,7-Bis(4,4,5,5-tetramethyl-1,3,2-dioxoborolan-2-yl)-9,9-dioctylfluorene (4) (150 mg, $0.23 \mathrm{mmol}), 1,4$-dihexyloxy-2,5-diiodobenzene (5) (121.8 $\mathrm{mg}, 0.2299 \mathrm{mmol})$ and monomer 3 $\left(0.119 \mathrm{mg}, 1.15 \times 10^{-4} \mathrm{mmol}\right)$ were used in the polymerization. GPC: $M_{n}=1.34 \times 10^{4}$, $\mathrm{PDI}=2.51$.

Since the content of naphthalimide dopant in polymers P1-P4 is very low, all these polymers showed similar ${ }^{1}$ H NMR spectra. Only P4 showed weak signals typical of the naphthalimide moiety. ${ }^{13} \mathrm{C}$ NMR spectra and elemental analysis results were similar for all these polymers as well. For example, for $\mathbf{P 1}:{ }^{1} \mathrm{H}$ NMR $\left(\mathrm{CDCl}_{3}, 200 \mathrm{MHz}\right) . \delta=7.82-7.57(\mathrm{~m}, 6 \mathrm{H}), 7.14(\mathrm{~s}, 2 \mathrm{H}), 3.99$ (bs, $\left.4 \mathrm{H},-\mathrm{OCH}_{2}{ }^{-}\right), 2.04$ (bs, $\left.4 \mathrm{H},-\mathrm{CH}_{2}{ }^{-}\right), 1.90-1.10\left(\mathrm{~m}, 4 \mathrm{OH},-\mathrm{CH}_{2}{ }^{-}\right) 0.88-0.79\left(\mathrm{~m}, 12 \mathrm{H},-\mathrm{CH}_{3}\right)$.

${ }^{13} \mathrm{C} \mathrm{NMR}\left(\mathrm{CDCl}_{3}, 75 \mathrm{MHz}\right) . \delta=150.59(\mathrm{C}=0), 150.50(\mathrm{C}=0), 139.89,137.05,131.26,127.95$, 124.52, 119.30, 116.77, $69.91\left(\mathrm{O}-\mathrm{CH}_{2}\right), 55.09\left(\mathrm{C}_{\text {bridge }}\right), 40.62\left(\mathrm{C}_{\text {bridge }}-\mathrm{CH}_{2}\right), 31.87,31.58,30.43$, $29.51,25.85,24.13,22.74,22.67,14.11,14.08$.

Anal. calcd. for $\mathrm{C}_{47} \mathrm{H}_{70} \mathrm{O}_{2}$ : C: $84.63 \%$; $\mathrm{H}: 10.58 \%$; Found C: $83.97 \%$; $\mathrm{H}: 10.08 \%$. 


\section{Results and discussion}

\subsection{Synthesis and characterization}

The synthetic approach followed for the synthesis of functionalized co-polymers P1-P4 with different content of naphthalimide and the reference polymer poly(fluorene-alt-phenylene) (PFP) is shown in Scheme 1. As it can be seen, it requires the preparation of a monomer (4) containing the selected napthalimide chromophore and appropriate functional groups which could enable its further co-polymerization, with fluorene monomer $\mathbf{5}$ and benzene derivative 6. The presence in these comonomers of long alkyl chains will make possible the synthesis of processable materials, which is a prerequisite for the fabrication of devices and the investigation of their optical properties in solution and solid phase, as discussed below. Also to enhance the solubility and processability we have chosen a long non-conjugated alkyl spacer between the poly(fluorene-alt-phenylene) backbone and the naphthalimide side groups. This is expected to improve the conformational flexibility of the side groups with respect to the chain, which has proven to be of interest regarding the photophysics of these materials [14].

Suzuki [32] couplings constitute one of the most convenient procedures for the preparation of polyarylenes, by the Pd-catalyzed cross-coupling reaction of aromatic diboron derivatives with aryl-dihalides in the presence of a base. This methodology has provided polyarylenes with high structural variety [33] and has enabled us the attachment of different electroactive functional moieties to conjugated polymers [25]. Besides, the compatibility of the reaction conditions with the imide functionality and the possibility to obtain structurally well-defined alternating fluorene-phenylene co-polymers has motivated us to choose this protocol for the preparation of co-polymers PFP and P1-P4.

Monomer 4 was synthesized from naphthalimide 2, prepared by heating the corresponding anhydride 1[34] with $p$-aminophenol in imidazole under the classical conditions [35]. This has enabled the synthesis of a versatile naphthalimide building block endowed with a strongly electron-donor diphenylamino group and a phenol functionality, which can be covalently linked to 3[13], a polymerizable diiodoarylene derivative bearing an additional complementary iodine reactive group. Thus, the Williamson etherification reaction between $\mathbf{3}$ and the complementarily functionalized naphtalimide $\mathbf{2}$ using potassium carbonate as the base in DMF afforded monomer 4 in a moderate $32 \%$ yield.

Monomer $\mathbf{4}$ has been characterized by the usual spectroscopic and analytical techniques. Thus, the ${ }^{1} \mathrm{H}$ NMR spectra of $\mathbf{4}$ shows the characteristic signals corresponding to the aromatic protons of both the naphthalimide and the naphthylphenylamine substituent at low fields, and signals for the alkyl chain at higher fields. On the other hand, the ${ }^{13} \mathrm{C}$ NMR spectrum shows, together with the carbonyl groups, the signals of the iodine-bearing aromatic carbons, the alkoxy groups and the alkyl spacer. The FTIR spectrum of monomer 4 further proves its structure and contains the typical strong bands at 1707 and $1668 \mathrm{~cm}^{-1}$ for imide groups and evidence concerning its purity is given by elemental analysis, which is in accordance with the expected values.

As it has been mentioned, co-polymers P1-P4 were synthesized by Suzuki polycondensations. Thus, mixtures with different content of the naphthalimide-containing monomer 4, 2,5-diiodo- 
1,4-dihexyloxybenzene (6) [28], and 2,7-bis(4,4,5,5-tetramethyl-1,3,2-dioxaborolan-2-yl)-9,9dioctylfluorene (5) [27] in the presence of $5 \mathrm{~mol} \% \mathrm{Pd}\left(\mathrm{PPh}_{3}\right)_{4}$ in a mixture of degassed THF and aqueous potassium carbonate was heated at reflux for $48 \mathrm{~h}$ (Scheme 1).

Although boronic acids have often been directly used as reagents in poly-Suzuki couplings, boronic esters like 5 have proven to be more advantageous in this kind of reactions, since the presence of the 1,1,2,2-tetramethylethylene glycol units has a protective effect on the labile boronic acid precursor. Simultaneously to the polymerization, a hydroxylation deprotection process takes place, in which the removal of such protecting groups as ethylene glycol does not affect the couplings [36]. The resulting alternating copolymers were then precipitated out of the thick reaction mixtures by addition of methanol, and soxlhet extracted with chloroform to separate from the insoluble fractions. Further purification of co-polymers P1-P4 was achieved by re-precipitation from dichloromethane/methanol several times and thorough washes with additional methanol and diethyl ether to remove ionic species and unreacted materials, to give yellow to orange solids in moderate to good yields. The starting 6:4 monomer ratios were adjusted in order to investigate the effect of polymer composition on the physical and optical properties of the final polymers. Thus, the content of 1,8naphthalimide in the resulting polymers was controlled in the range $0.05-8 \mathrm{~mol} \%$. As it will be discussed below, this low concentration of naphthalimide is enough by itself to modify the optical properties of co-polymers P1-P4 with respect to the parent non-substituted reference PFP.

In order to be used as a reference material, we have also synthesized co-polymer poly[9,9dioctyl-9H-fluorene-2,7-diyl][2,5-bis(hexyloxy)-1,4-phenylene] (PFP, $x=0$ in Scheme 1), containing the same polymeric backbone as P1-P4 but without pendant naphthalimide units, by an analogous Suzuki polycondensation between 1,4-dihexyloxy-2,5-diiodobenzene (6) and bisborolane 5 under the same conditions employed in the synthesis of P1-P4. Also for comparison, we synthesized as reference material the 1,8-naphtalimide derivative 7[27] as shown in Scheme 2.

The alkyl substituents in the main chain of co-polymers P1-P4 provide these materials good solubility in common organic solvents, such as toluene, chloroform or THF, and enabled their full characterization by NMR and electrochemical and optical techniques.

In spite of the increasing naphthalimide dopant content, all polymers P1-P4 showed similar ${ }^{1} \mathrm{H}$ NMR patterns. A multiplet corresponding to the six fluorene aromatic protons is visible at around 7.8-7.6 ppm and the singlet of the two protons on the benzene ring is seen at around $7.14 \mathrm{ppm}$. The characteristic signals of the alkoxy chains appear in the expected region (ca. $4.0 \mathrm{ppm}$ ) and the ${ }^{1} \mathrm{H}$ NMR spectra is completed with the signals of the alkyl chains at lower fields, including at slightly higher shifts those of the methylenes directly linked to the fluorene carbon bridge at around $2.0 \mathrm{ppm}$. Only P4, the functionalized polymer with the highest content of naphthalimide, showed very weak multiplets that could be assignated by comparison to the pendant naphthalimide groups. For P4, integration of the weak signals between 8.59 and $8.25 \mathrm{ppm}$ corresponding to the naphthalimide group and those diagnostic signals corresponding to the aromatic backbone indicate a $0.08 \%$ of naphthalimide units in the 
polymer. This corresponds to the percent of naphthalimide-containing monomer added in the reaction and provides evidence of the similar reactivity of monomers $\mathbf{4}$ and $\mathbf{6}$.

Gel permeation chromatography (GPC) with polystyrene as standard revealed that the copolymers P1-P4 have similar molecular weights $\left(\overline{M_{n}}\right)$, between 14,400 and $13,400 \mathrm{~g} / \mathrm{mol}$, with polydispersities (PD) ranging from 2.51 to 3.26 (Table 1). The molecular weights, without considering the known overestimation based on PS calibration [37] and considering the $M_{\mathrm{w}}$ as major weight provides polymer lengths between 70 (P4) and 50 (P1) units based on a molecular unity of $664 \mathrm{~g} / \mathrm{mol}$. The lower values of molecular weights for co-polymers P1-P4 in comparison with the reference PFP (75 units) suggest that the presence of the lateral chromophore units affects slightly the polymerization process. Nevertheless, the electrochemical and photophysical properties of the polymers are not expected to be influenced by this slight differences in the polymer length considering that all of them exceed by far the effective conjugation length.

\subsection{Electrochemical properties}

The redox properties of novel polymers P1-P4 and PFP were determined by cyclic voltammetry at room temperature in dichloromethane solutions, using a platinum disk and wire as working and counter electrodes, respectively, $\mathrm{Ag} / \mathrm{AgCl}$ as reference electrode, and tetrabutylammonium hexafluorophosphate (TBAHFP, $0.1 \mathrm{M}$ ) as supporting electrolyte.

The cyclic voltammograms of polymers P1-P4 show the same redox behaviour as PFP (Fig. 2), presenting a relatively broad quasireversible wave with an onset oxidation potential at around $+0.53 \mathrm{~V}$ (vs. $\mathrm{Fc} / \mathrm{Fc}^{+}$) assigned to the reversible $p$-doping processes of the poly(fluorenealt-phenylene) conjugated chain. No reduction processes on the polymeric backbone could be detected up to $-2.5 \mathrm{~V}$ under our experimental conditions [38]. This result indicates that these concentrations of naphthalimide units lead to little change in the electrochemical properties of the resulting polymers. Naphthalimide reference $\mathbf{7}$ showed a sharp and well resolved quasireversible reduction wave at around $-1.80 \mathrm{~V}$, evidencing the moderate electron acceptor ability of this moiety due to the presence of the electron-rich diarylamino substituent. We have observed for this type of functionalized polymers that both electroactive units, the electron donor conjugated backbone and the electron acceptor naphthalimide units preserve their nature on the ground state [8], [12] and [13]. Consequently, no electron transfer processes are expected in the ground state.

\subsection{Photophysical properties}

The UV-vis absorption spectra of co-polymers P1-P4 and PFP in dichloromethane solutions together with the excitation spectrum of the reference $\mathbf{7}$ in chloroform solution are depicted in Fig. 3a. Functionalized co-polymers P1-P4 exhibit the same absorption spectra as PFP, with a maximum at $375 \mathrm{~nm}$ which can be attributed to the $\pi-\pi *$ transition of the conjugated poly(fluorene-alt-phenylene) backbone. As it was the case in the electrochemical investigation, the low naphthalimide chromophore content does not produce any noticiable change on the absorption properties of these materials. Only a residual absorption from the pendant chromophore, peaking at around $469 \mathrm{~nm}$ in reference 7 , could be observed, confirming the minimal interaction between the chromophores in the ground state. 
Fig. $3 b$ shows the absorption spectra of the P1-P4 and PFP thin films together with a blend film with the same polymer/naphthalimide ratio as in P4 (i.e. 0.08), which are very similar to that in solution. Again, there is no observable difference between the copolymers and the PFP absorption spectra and only a very weak contribution of the band around $466 \mathrm{~nm}$ corresponding to the naphthalimide chromophore $\mathbf{7}$ could be detected. Nevertheless, for the blend thin film, the absorption maximum is slightly red shifted with respect to the co-polymers and shows a larger contribution in the naphthalymide chromophore $\mathbf{7}$ absorption region. The optical gap for the thin films, estimated from the departure of the OA derivative from zero, does not experiment important changes: 2.87 and $2.81 \mathrm{eV}$ for the co-polymers and the Blend film, respectively.

Fig. 4 shows the emission spectra in diluted $\left(c a .10^{-5} \mathrm{M}\right)$ chloroform solutions of the P1-P4 copolymers, a solution mixture with the same polymer/naphthalimide ratio as in $\mathbf{P 4}$ (recorded all of them with excitation at $370 \mathrm{~nm}$ ) and the PFP and naphthalimide 7 for reference. For the sake of clarity, we present the $560-790 \mathrm{~nm}$ region magnified to appreciate the emission of the solutions in Fig. 4b. PFP emission shows their known emission peaking at $420 \mathrm{~nm}$ [13]. The naphthalimide reference 7 presents its maximum at $622 \mathrm{~nm}$.

The co-polymers and the mixture in diluted solutions present the same blue emission of PFP, with maximum at $420 \mathrm{~nm}$, together with a small emission associated with the naphthalimide pendant group peaking at $622 \mathrm{~nm}$ (except for $\mathbf{P} \mathbf{1}$ thin film). No changes in the bands are observed except for a quenching of the blue emission $(420 \mathrm{~nm})$ when increasing the naphthalimide chromophore concentration and a small increase of the $622 \mathrm{~nm}$ band with increasing cromophore content.

Although this $622 \mathrm{~nm}$ band of the spectrum is not very strong in comparison with the blue part of the spectra, it can be seen in Fig. $4 \mathrm{~b}$ that the red band intensity is higher for the P4 copolymer, with the pendant group covalently attached, than for the mixture, indicating that the energy transfer is more efficient in the co-polymers than in the mixture prepared with PFP and the reference $\mathbf{7}$, with the same ratio as in $\mathbf{P 4}$. This can be understood since the energy transfer between donor and acceptor chromophores depends on the intermolecular distance between the donor and acceptor molecules which is favoured in the covalently attached co-polymers compared to the mixture of components in solution.

Thus, the incorporation of the 1,8-naphtalimide derivative as side chain to poly(fluorene-altphenylene) leads to negligible changes in the solution UV-vis absorption spectra but results in a quenching of the PFP emission and the more effective emission of the $622 \mathrm{~nm}$ band in the solution PL spectra of these co-polymers. This can be explained by a Föster energy transfer from the polymer backbone to the naphthalimide moiety unit as a consequence of the overlap between the absorption spectrum of the naphthalimide and the PFP backbone emission spectrum. Moreover, since the absorbance is greater for the PFP backbone than for the naphtalimide chromophore (Fig. 3a) at the excitation wavelength for PL ( $370 \mathrm{~nm}$ ), nearly all the incident photons are absorbed by the PFP main chain, indicating that this Föster energy transfer is very efficient. With the increase of the content of naphtalimide, the contribution of the $622 \mathrm{~nm}$ emission in Fig. 4 becomes larger in the co-polymers due to the completion of the energy transfer which is less efficient in the physical blend. 
Environmental Scanning Electron Microscopy (ESEM) images (Fig. 5) of P4 and a Blend film prepared with the same polymer/naphthalimide ratio as in $\mathbf{P 4}$ have been taken in backscattering detector configuration. As it can be observed, both films present a very good uniformity but we find islands with larger atomic weight for the Blend film that could be associated to naphthalimide aggregates, not observed in the $\mathbf{P 4}$ co-polymer film. This has been as well confirmed by the larger amount of carbon obtained by microanalysis (Energy Dispersion X Ray Spectrometry (EDX)). This moves us to assume that the naphthalimide chromophore $\mathbf{7}$ is more uniformly distributed in the co-polymer than in the blend film. Those islands could be responsible for the low energy band observed in the absorption spectrum of the blend film.

With respect to the emission in the solid state (Fig. 6), the PFP emission spectrum exhibits the same shape as in solution, slightly red sifted, with emission maximum at $422 \mathrm{~nm}$. However, in P1-P4 copolymers and Blend thin films the blue band almost vanishes when increasing the naphthalimide content and the spectra are dominated by an orange broad band (500-650 nm) not observed in solution. This orange band peaks at $524 \mathrm{~nm}$ for $\mathbf{P} \mathbf{1}(x=0.0005)$ and shifts to the red with the increasing concentration of orange chromophore to $549 \mathrm{~nm}$ for P4 $(x=0.08)$. Blend thin film presents mainly the same spectrum as $\mathbf{P 4}$ only slightly red shifted. The inset of Fig. 6 shows the Gaussian deconvolution of the naphthalimide chromophore $\mathbf{7}$ diluted solution emission which fits well to three components at $1.807 \mathrm{eV}(687 \mathrm{~nm}), 2.015 \mathrm{eV}(616 \mathrm{~nm})$ and $2.296 \mathrm{eV}(542 \mathrm{~nm})$. We can observe that the weak component at $542 \mathrm{~nm}$ is drastically enhanced in the co-polymers thin film emission. We also observe that the energy transfer has a stronger effect in thin films than in solution owing to stronger intermolecular interactions in solid state [39]. The photographs of PFP and P4 thin films under UV illumination are shown in Fig. 6. We observe a light blue to the eye for the PFP co-polymer film and a yellow-white to the eye color luminescence emission for the P4 co-polymer film.

Fig. 7 shows the PL decay curves for PFP, P2, P3, P4 and Blend thin films on quartz substrates exciting at $380 \mathrm{~nm}$. All the analyzed samples show single-exponential decay which is indicative of only one emitting species and thus cannot be associated to excimers or aggregates [40]. The $450 \mathrm{~nm}$ emission of FPF thin film presents a simple exponential decay with time constant ( $\tau_{\text {blue }}$ ) of $7 \mathrm{~ns}$. The time constants of P2, P3 and $\mathbf{P 4}$ emission at $530 \mathrm{~nm}\left(\tau_{530}\right)$ are the same, slightly increasing with the naphtalimide concentration, 13.4, 14.5 and $14.9 \mathrm{~ns}$, respectively. P4 also exhibits a significant emission in the red at $600 \mathrm{~nm}$ with $\tau_{600}=14.4 \mathrm{~ns}$, very similar to $530 \mathrm{~nm}$ emission life time in the same sample. The physical Blend (not shown in the graph) exhibits the same lifetimes as P4. Thus, the present PL lifetime data together with the steady PL results, provide evidence that the orange emission in co-polymers could not be associated to FPF, as $\tau_{530}$ is twice $\tau_{\text {blue, }}$ and the centre responsible is the same in all co-polymers. This orange band arises from a centre associated to the naphtalimide chromophore significantly enhanced in solid state.

We have calculated the thin films PL quantum yields, $\phi_{\mathrm{PL}}$, as described in the experimental part. We have observed that the lateral introduction of the naphthalimide diminishes drastically the emission efficiency from 0.89 for PFP to $0.39,0.17,0.41,0.38$ and 0.40 for P1P4 and Blend thin films, respectively. The lowest $\phi_{\mathrm{PL}}$ corresponds to $\mathbf{P 2}$ while the other copolymers (P1, P3 and $\mathbf{P 4}$ ) and the Blend thin films show similar values of $\sim 0.4$. One possible 
reason for the decrease in the PL quantum yield may be the presence of a competitive photoinduced electron transfer (PET) process. In this regard, by using the Weller equation [41], it has been calculated that the PET process is energetically favourable as the corresponding $\Delta G_{\mathrm{PET}}$ was estimated to be $-0.42 \mathrm{eV}$ in dichloromethane [42]. The PL quantum yields, together with the charge injection efficiency [43] and the intrinsic transport properties of the copolymers, have a great influence in the electroluminescence efficiency of the devices as it is proportional to PL quantum yield.

\subsection{Electrical measurements}

Simple OLED structures were fabricated according to the method described in the experimental section, with configuration: ITO/PEDOT:PSS (50 nm)/polymer (90-125 nm)/Ba/AI $(200 \mathrm{~nm})$, in order to investigate the electroluminescent (EL) properties of the materials. Fig. 8 shows the $I-V$ characteristic for the diodes based on P1 to P4 copolymers together with a scheme of the energy levels of the devices based on these materials. The $\mathbf{P 2}$ based diode, exhibits the worse performance with a threshold voltage at around $\mathbf{9} \mathbf{V}$. In P3 and P4 based diodes, the threshold voltage decrease to 7 and $5.5 \mathrm{~V}$, respectively. The P1 based diode shows a very similar performance to $\mathbf{P 4}$, with threshold voltage of $5.5 \mathrm{~V}$. Unfortunately, the correlation between the $I-V$ threshold voltage and the naphthalimide content is obscured by the dispersion in the active layer thicknesses.

The EL emissions are presented in Fig. 9 together with the driving currents, applied voltage and $\mathrm{CIE}$ color coordinates obtained for each device. It has to be noted that all the devices exhibited emission in the $\mu \mathrm{A}$ range: $47,73,39,60 \mu \mathrm{A}$ for $\mathbf{P 1}, \mathbf{P 2}, \mathbf{P} \mathbf{3}$ and $\mathbf{P 4}$ based devices, respectively. The efficiencies obtained are 22.62, 7.53, 47.11 and 2.93 Cd/A for P1, P2, P3 and P4 based devices, respectively.

A good match between the PL and EL spectra is observed, indicating that both emissions are produced by the same species. Although in the EL spectra, a larger contribution of the orange band is observed. This is attributed to the additional charge-trapping effect of the 1,8naphthalimide which will act as a charge-trapping centre during the electron transport process, as described in organic host/guest systems [44]. This will be an additional contribution to the orange emission apart from the Föster energy transfer from the polymer backbone to the 1,8-naphthalimide chromophore which corresponds to that observed in the PL measurements. Indeed, the orange broad band is the bigger contribution in all the spectra and the blue emission is only observed for $\mathbf{P} \mathbf{1}$ and residually for $\mathbf{P} \mathbf{2}$ based devices. $\mathbf{P} \mathbf{3}$ and $\mathbf{P 4}$ based devices exhibit only the broad orange contribution, as observed in the thin films PL emission. We observe white emission $(0.30,0.42)$ for the $\mathbf{P} \mathbf{1}$ based device, where the blue and orange emission is more balanced.

\section{Summary}

A series of single polymers exhibiting simultaneous blue and orange emission in solid state that gives rise to white emission have been synthesized and characterized. Photophysical studies suggest that this broad band is associated with an efficient energy transfer from the PFP backbone to the naphthalimide orange chromophore. Although energy transfer may be observed in diluted solutions, solid state drastically enhances it due to a increasing of 
intermolecular interactions that gives rise to a more efficient excitation energy transfer from the blue emission of the PFP backbone to the naphthalimide chromophore. This energy transfer is more effective in co-polymers than for physical mixtures of the two chromophores. White light $(0.30,0.42)$ is obtained from a single layer, single component WOLED with a efficiency of $22.62 \mathrm{Cd} / \mathrm{A}$.

\section{Acknowledgement}

We thank Comunidad Autónoma de Madrid (Project S2009/MAT-1756 and S2009/MAT-1467), the UCM-BSCH Joint Project (GR58/08), the CAM-UCM Joint Project (CCG08-UCM-PPQ-3957) and the MCYT of Spain (MAT2009-08786, CTQ2007-60459) for financial support. We also thank to G. del Rosario of URJC-CAT services for the ESEM discussion. R.B, R.J and A.P. are indebted, respectively, to the "Comunidad de Madrid" the "Universidad Rey Juan Carlos" and "Universidad Complutense" for predoctoral fellowships.

\section{References}

[1] (a) McQuade DT, Pullen A, Swager ETM. Chem Rev 2000;100:2537; (b) Thomas III SW, Joly GD, Swager TM. Chem Rev 2007;107:1339.

[2] Gómez R, Segura JL. In: Nalwa HS, editor. Handbook of organic electronic and photonics. Los Angeles: American Scientific Publishers; 2007.

[3] Gunes S, Neugebauer H, Sariciftci NS. Chem Rev 2007;107:1324.

[4] Roncali J. Chem Soc Rev 2005;34:483.

[5] Ramos AM, Rispens MT, van Duren JKJ, Hummelen JC, Janssen RAJ. J Am Chem Soc 2001;123:6714.

[6] Zerza G, Cravino A, Neugebauer H, Sariciftci NS, Gomez R, Segura JL, et al. J Phys Chem A 2001;105:4172.

[7] Giacalone F, Segura JL, Martín N, Catellani M, Luzzati S, Lupsac N. Org Lett 2003;5:1669.

[8] Segura JL, Gómez R, Blanco R, Reinold E, Bäuerle P. Chem Mater 2006;18:2834.

[9] (a) Blanco R, Seoane C, Segura JL. Tetrahedron Lett 2008;49:2056; (b) Gómez R, Blanco R, Veldman D, Segura JL, Janssen RAJ. J Phys Chem B 2008;112:4953.

[10] Catellani M, Luzzati S, Lupsac NO, Mendichi R, Consonni R, Famulari A, et al. J Mater Chem 2004;14:67.

[11] Luzzati S, Scharber M, Catellani M, Giacalone F, Segura JL, Martín N, et al. J Phys Chem B 2006;110:5351.

[12] Segura JL, Gómez R, Reinold E, Bäuerle P. Org Lett 2005;7:2345. 
[13] Gómez R, Veldman D, Blanco R, Seoane C, Segura JL, Janssen RAJ. Macromolecules 2007;40:2760.

[14] Montilla F, Esquembre R, Gómez R, Blanco R, Segura JL. J Phys Chem C 2008;112:16668.

[15] (a) Tu GL, Zhou QG, Cheng YX, Wang LX, Ma DG, Jing XB, et al. Appl Phys Lett 2004;85:2172; (b) Tu GL, Mei CY, Zhou QG, Cheng YX, Wang LX, Ma DG, et al. Adv Funct Mater 2006;16:101.

[16] (a) Liu J, Zhou QG, Cheng YX, Geng YH, Wang LX, Ma DG, et al. Adv Funct Mater 2006;16:957; (b) Liu J, Min C, Zhou QG, Cheng YX, Wang L, Ma D, et al. Appl Phys Lett 2006;88.

[17] Liu J, Guo X, Bu L, Xie Z, Cheng Y, Geng Y, et al. Adv Funct Mater 2007;17:1917.

[18] (a) Liu J, Zhou Q, Cheng Y, Geng Y, Wang L, Ma D, et al. Adv Mater 2005;17:2974; (b) Tu G, Mei C, Zhou Q, Geng Y, Wang L, Ma D, et al. Adv Funct Mater 2006;16:101.

[19] Lee SK, Ahn T, Cho NS, Lee J-I, Jung YK, Lee J, et al. Polym Sci Part A Polym Chem 2007;45:1199.

[20] <http://www1.eere.energy.gov/buildings/ssl/>.

[21] (a) D'Andrade BW, Forrest SR. Adv Mater 2004;16:1585; (b) Wang YZ, Sun RG, Meghdadi F, Leising G, Epstein AJ. Appl Phys Lett 1999;74:3613.

[22] Strukelj M, Jordan RH, Dodabalapur P. J Am Chem Soc 1996;118:1213.

[23] Tasch S, List EJW, Ekströn O, Graupner W, Leising G, Schlichting P, et al. Appl Phys Lett 2004;85:4076.

[24] (a) Gong X, Wang S, Moses D, Bazan GC, Heeger AJ. Adv Mater 2005;17:2053; (b) Gong X, Ma W, Ostrowski JC, Bazan GC, Moses D, Heeger AJ. Adv Mater 2004;16:615.

[25] Blanco R, Seoane C, Segura JL. Tetrahedron Lett 2008;49:2056.

[26] Jiang W, Yueming S, Wang X, Wang Q, Xu W. Dyes Pigments 2008;77:125.

[27] Dudek SP, Pouderoijen M, Abbel R, Schenning AHP, Meijer EW. J Am Chem Soc 2005;127:11763.

[28] Wang C, Batsanov AS, Bryce MR, Sage I. Org Lett 2004;6:2181.

[29] Lee J, Cho HJ, Cho NS, Hwang D-H, Kang J-M, Lim E, et al. J Polym Sci Part A Polym Chem 2006;44:2943; (a) Thomas III SW, Swager TM. Macromolecules 2005;38:2716.

[30] Coya C, de Andrés A, Gómez R, Seoane C, Segura JL. On the blue emission of a novel solution processed stilbenoid dendrimer thin film for OLED displays. J Lum 2008;128:761.

[31] Birks JB. Photophysics of aromatic molecules. New York, NY: Wiley; 1970.

[32] (a) Selected references on the Suzuki coupling: Suzuki A. Chem Commun 2005;38:4759; (b) Suzuki A. Proc Jpn Ac 2004;80:359; (c) Nicolau KC, Bulger PG, Sarlah D. Angew Chem Int Ed 
2005;44:4442; (d) Li CJ. Chem Rev 2005;105:3095; (e) Miyaura N, Yanagi T, Suzuki A. Synth Commun 1981;11:513; (f) Wallow TI, Novak BM. J Am Chem Soc 1991;113:7411; (g) Suzuki A. Acc Chem Res 1982;15:178.

[33] (a) Babudri F, Farinola GM, Naso F. J Mater Chem 2004;14:11; (b) Sakamoto J, Rehahn M, Wegner G, Schlüter AD. Macromol Rapid Commun 2009;30:653.

[34] Islam A, Cheng C, Chi S, Lee S, Hela PG, Cheng C. J Phys Chem B 2005;109:5509.

[35] (a) Xu Z, Xiao Y, Quian X, Cui J, Cui D. Org Lett 2005;7:889; (b) Gunnlaugsson T, Lee TC, Parkesh R. Org Biomol Chem 2003;1:3265; (c) Griesbeck AG, Schieffer S. Photochem Photobiol Sci 2003;2:113.

[36] (a) Kowitz C, Wegner G. Tetrahedron 1996;53:15553; (b) Goodson FE, Novak BM. Macromolecules 1997;30:6047.

[37] Molina R, Gómez-Ruiz S, Montilla F, Salinas-Castillo A, Fernández-Arroyo S, Ramos MM, et al. Macromolecules 2009;42:5471.

[38] PFP is reported to have an oxidation potential of $1.47 \mathrm{eV}$ and a reduction potential of $2.73 \mathrm{~V}$ vs. $\mathrm{Ag} / \mathrm{Ag}+$ in thin film cyclic voltammetry, Ranger $M$, Leclerc $M$, Can J Chem 1998;76:1571.

[39] Cacialli F, Friend RH, Bouché C-M, Le Bamy P, Facoetti H, Soyer F, et al. J Appl Phys 1998;83:2343.

[40] Valeur B. Molecular fluorescence. Principles and applications. Weinheim, Germany: WileyVCH; 2002.

[41] Weller A. Z Phys Chem 1982;133:93.

[42]

[43] Friend RH, Gymer RW, Holmes AB, Burroughes JH, Marks RN, Taliani C, et al. Nature 1999;397:121.

[44] Tasxch S, List EJE, Hockfilzer C, Leisisng G, Schlichting P, Rogr U, et al. Phys Rev B $1997 ; 56: 4479$ 
Figures, schemes and tables:

Fig. 1. Chemical structure of co-polymers P1-P4.

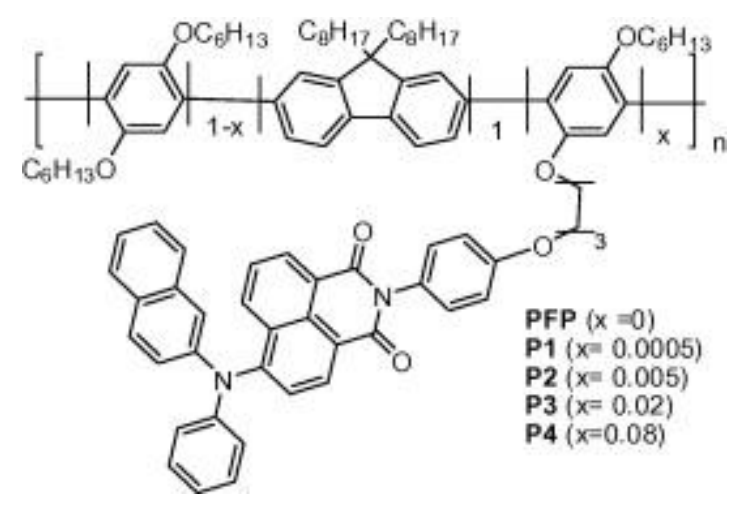


Scheme 1.(a) Synthesis of comonomer $\mathbf{4}$ and (b) Synthesis of co-polymers P1-P4 ( $x=$ molar ratio in feed).

(a)
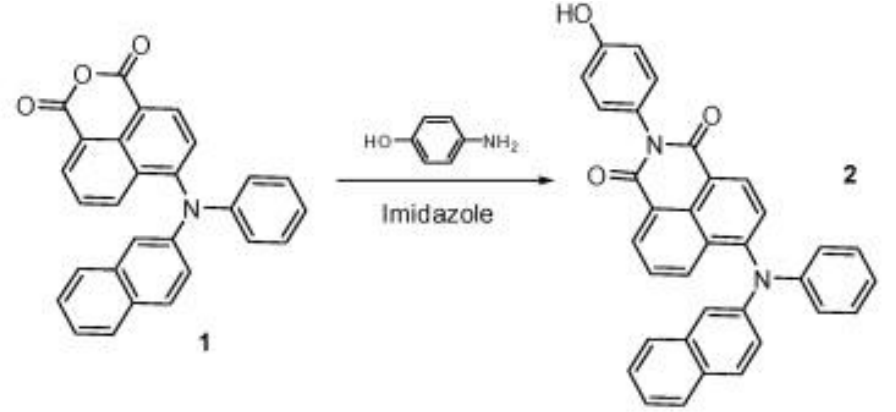

(b)
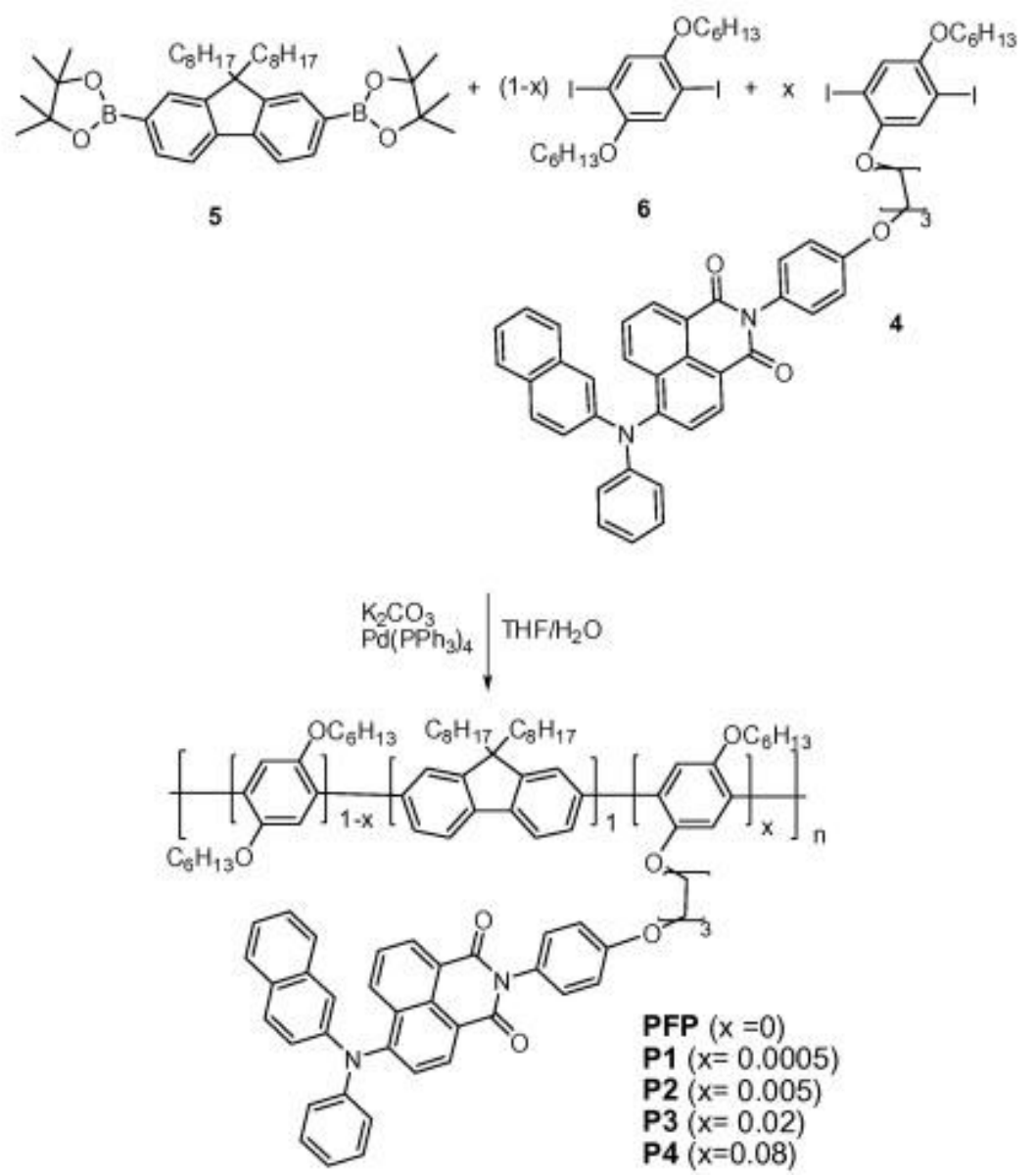
Scheme 2. Synthesis of reference material 7.
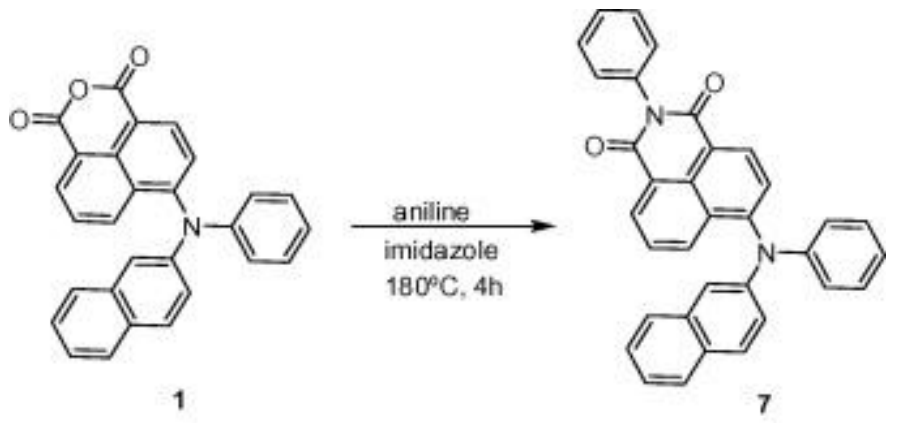

Table 1. Physical properties of the co-polymers.

\begin{tabular}{llll}
\hline Comp. & $\overline{M_{n}}{ }^{\mathrm{a}}$ & $\overline{M_{w}}{ }^{\mathrm{a}}$ & $\mathrm{PD}^{\mathrm{a}}$ \\
\hline PFP & 20,300 & 50,100 & 2.47 \\
P4 & 14,400 & 46,900 & 3.26 \\
P3 & 14,500 & 39,800 & 2.75 \\
P2 & 14,100 & 36,900 & 2.62 \\
P1 & 13,400 & 33,600 & 2.51 \\
\hline
\end{tabular}

a $\overline{M_{n}}, \overline{M_{w}}$ and PD of the polymers were determined by GPC with polystyrene standards in 1,2,4,5-tetrachlorobenzene (TCB) as solvent.

Fig. 2. Cyclic voltammograms of polymers P1-P4, and PFP and 7 references in dichloromethane/TBAHFP $(0.1 \mathrm{M})$ at a scan rate of $100 \mathrm{mV} \mathrm{s}^{-1}$. All measurements were carried out at room temperature and potentials are given vs. $\mathrm{Fc} / \mathrm{Fc}^{+}$.

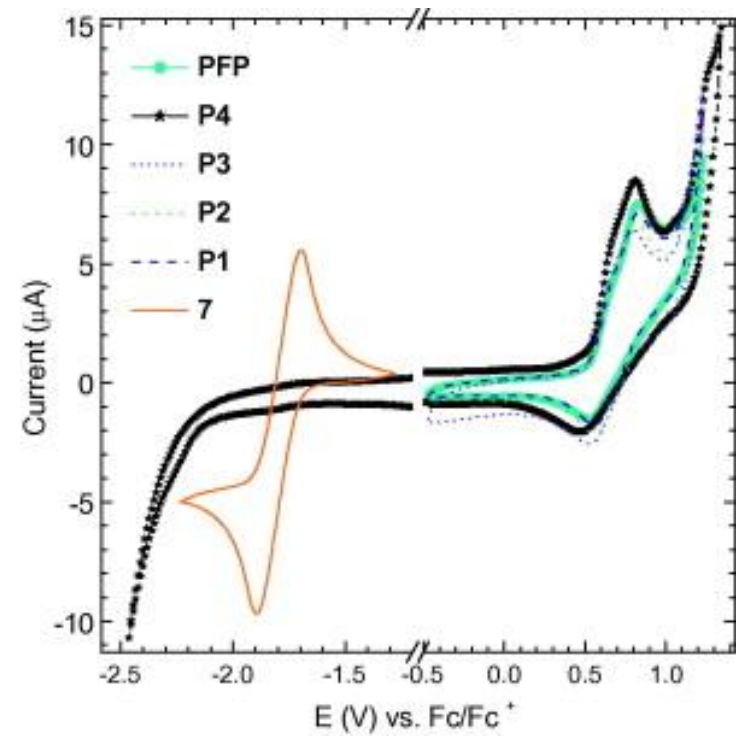


Fig. 3. (a) UV-vis absorption spectra of co-polymers P1-P4 and PFP in dichloromethane solutions, together with the excitation spectrum of 7 in a $10^{-5} \mathrm{M}$ chloroform solution. (b) UVVis absorption spectra of co-polymers P1-P4, PFP and $\mathbf{7}$ thin films together with that of a thin film of the physical blend between PFP and the naphthalimide reference $\mathbf{7}$ fabricated with the same polymer/naphthalimide ratio as in P4 (i.e. 0.08).

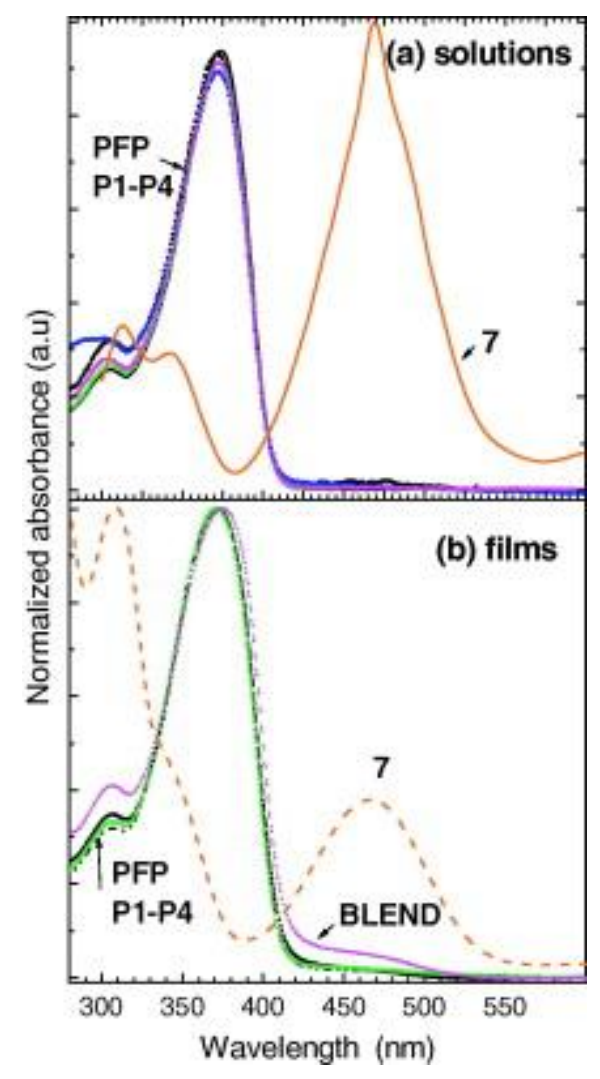

Fig. 4. Emission spectra of PFP, P1-P4, 7 and a solution mixture (Blend) with the same polymer/naphthalimide ratio as in P4 (i.e. 0.08). Spectra recorded with excitation at $370 \mathrm{~nm}$ in diluted $\left(\mathrm{ca} .10^{-5} \mathrm{M}\right)$ chloroform solutions.

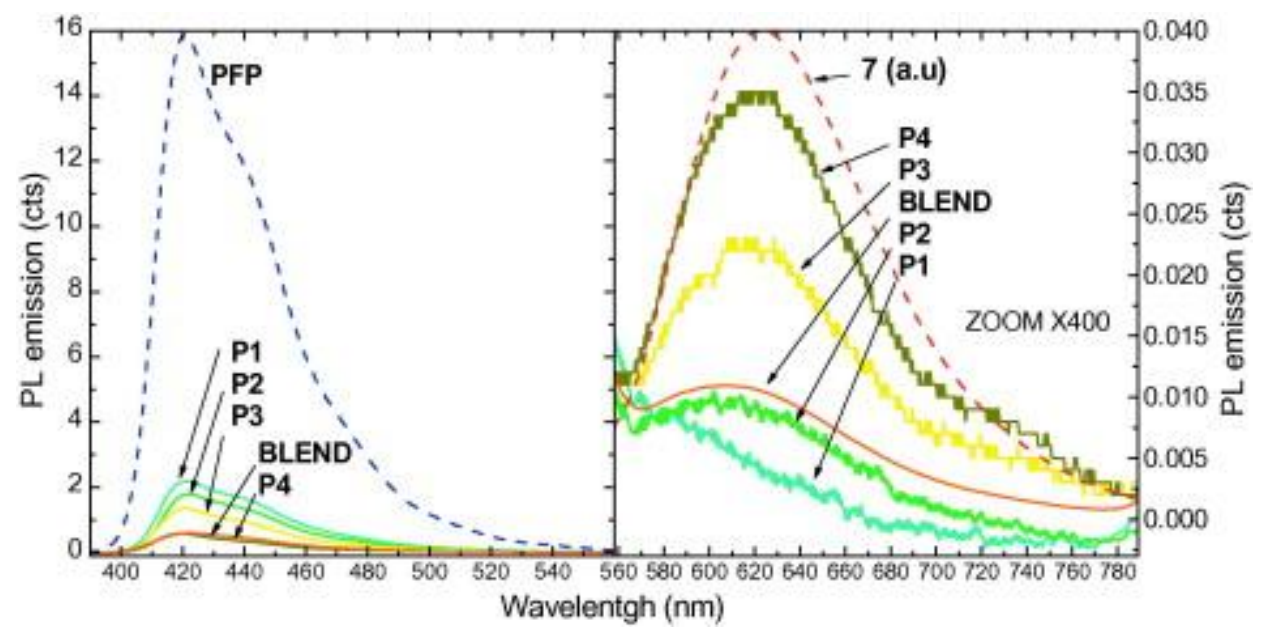


Fig. 5. ESEM images in backscattering detector configuration of blend (a) and P4 (b) thin films.

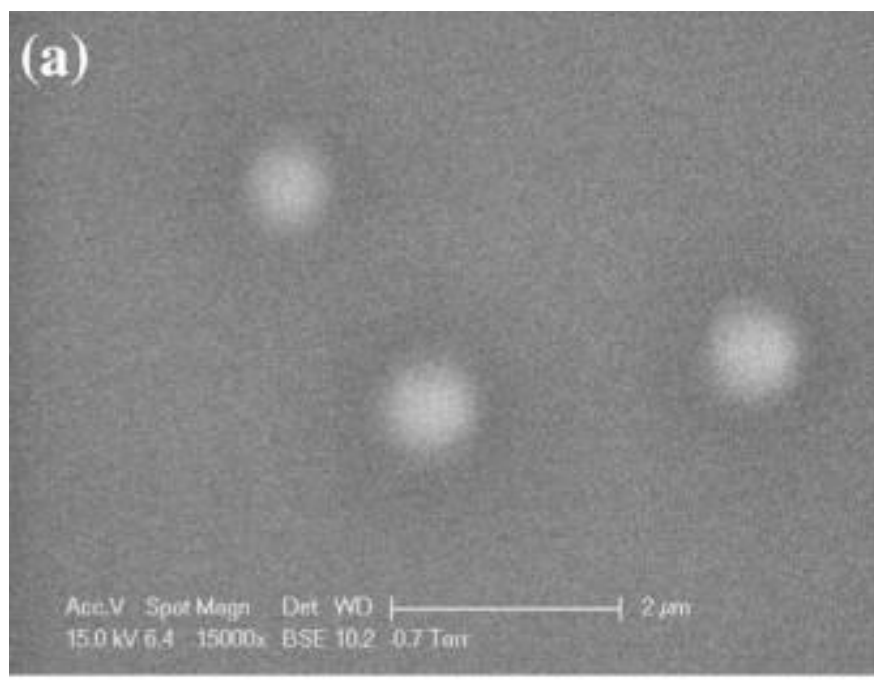

(b)

Ace.V Spot Magn. Det wo

$15.0 \mathrm{KV} 5.8$ 15000x BSE 10.7 0.7 Tar 
Fig. 6. Emission spectra of thin films of P1-P4, PFP and a Blend prepared with the same polymer/naphthalimide ratio as in P4 (i.e. 0.08). The inset shows the PL emission normalized relative to the blue emission at $422 \mathrm{~nm}$, as indicated by the arrow. Spectra recorded with excitation at $370 \mathrm{~nm}$. In the insets, photographs of the PFP and P4 films under UV (291 nm) illumination are depicted.

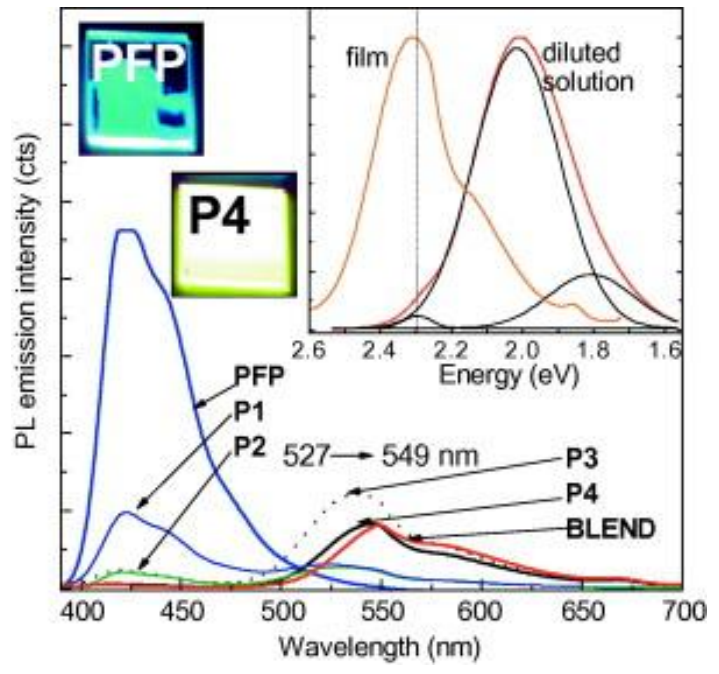


Fig. 7. PL decay of the thin films measured with detector at 440, 530 and $600 \mathrm{~nm}$, all excited at $380 \mathrm{~nm}$. Open symbols represents the experimental data and solid lines are single-exponential fits to the data.

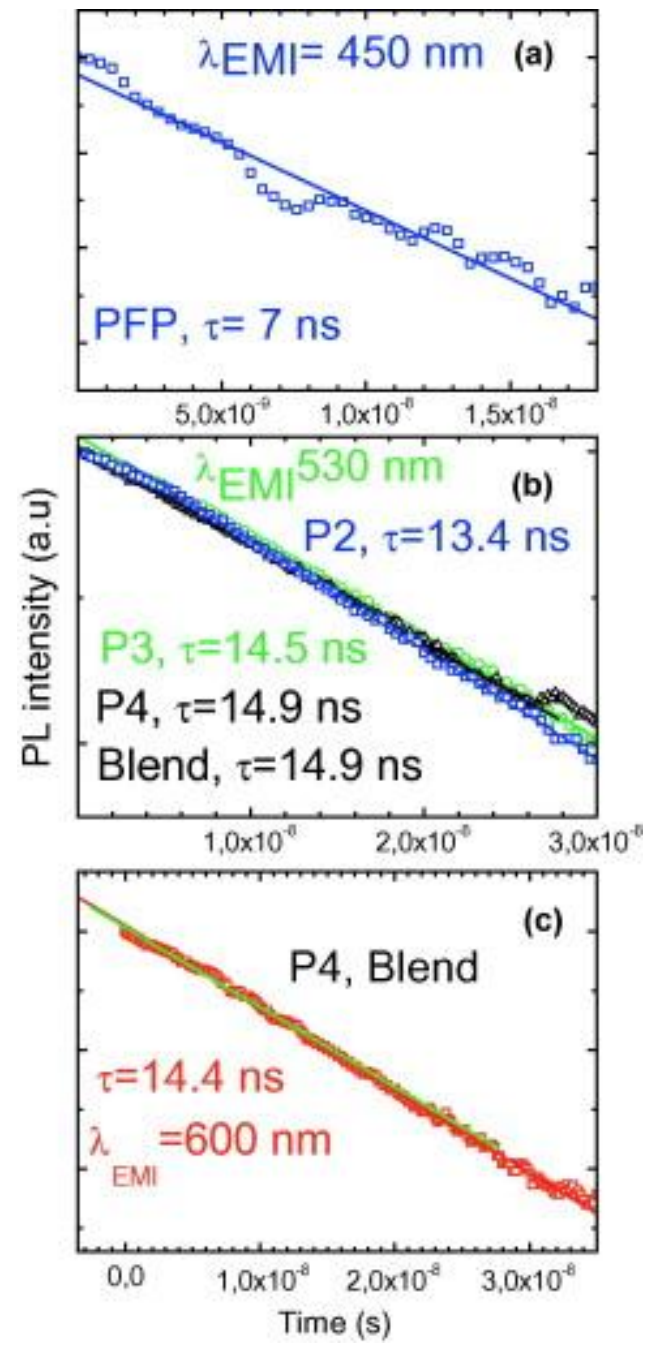

Fig. 8. Experimental $I-V$ curves of the OLED based on ITO/PEDOT: PSS/active layer/BaAl. Schematic representation of the electronic levels of the material in the inset.

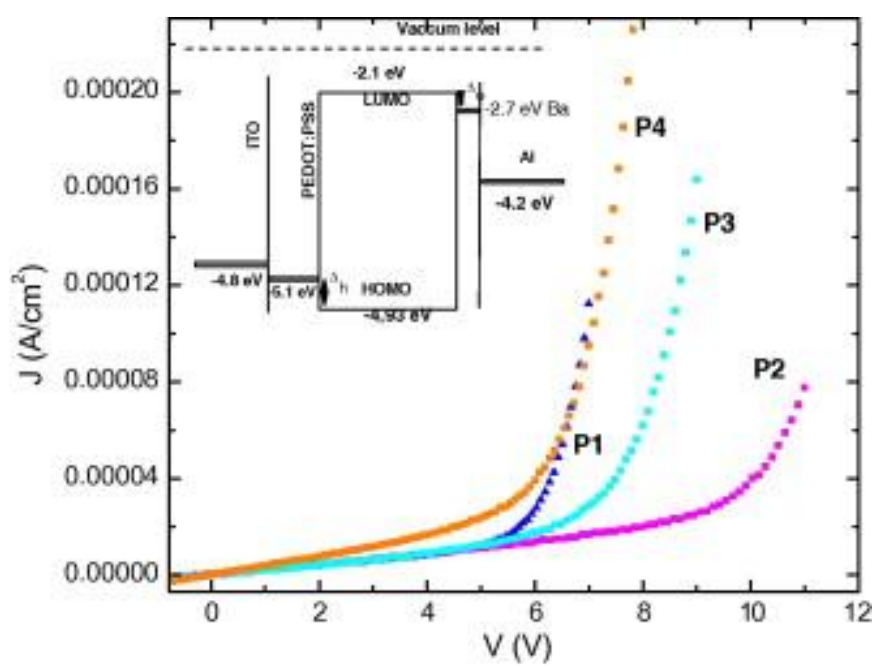


Fig. 9. Electroluminescence emission of the devices fabricated with P1, P2, P3 and P4 copolymers as active layers.

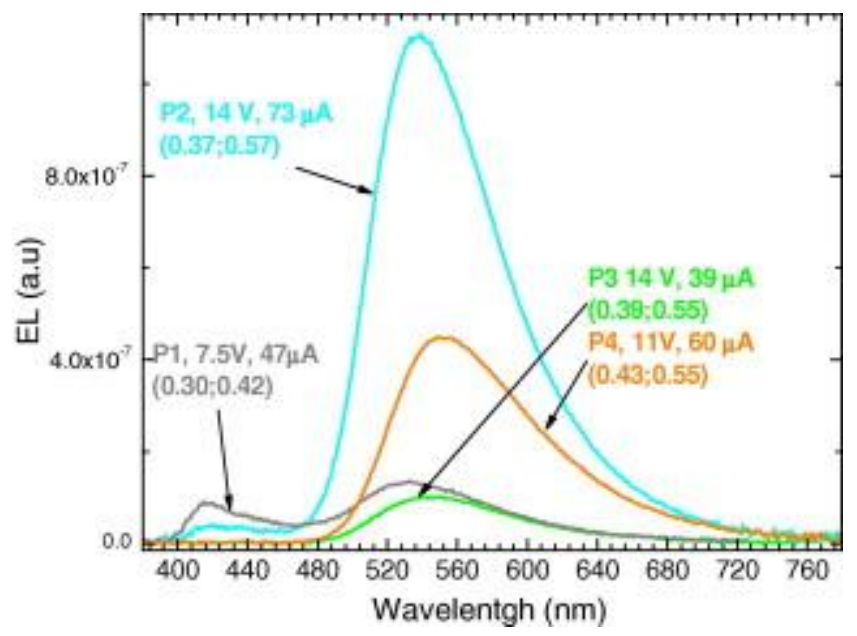

